\title{
Conflict Minerals from the Democratic Republic of the Congo: Global Tungsten Processing Plants, a Critical Part of the Tungsten Supply Chain
}

The U.S. Geological Survey (USGS) analyzes supply chains to identify and define major components of mineral and material flows from ore extraction, through intermediate forms, to a final product. Two major reasons necessitate these analyses: (1) to identify risks associated with the supply of critical and strategic minerals to the United States and (2) to provide greater supply chain transparency so that policymakers have the information necessary to ensure domestic legislation compliance. This fact sheet focuses on the latter. The USGS National Minerals Information Center has been asked by governmental and non-governmental organizations to provide information on tin, tantalum, tungsten, and gold (collectively known as "3TG minerals") processing facilities worldwide in response to U.S. legislation aimed at removing the link between the trade in these minerals and civil unrest in the Democratic Republic of the Congo.

Post beneficiation processing plants (smelters and refineries) of $3 \mathrm{TG}$ mineral ores and concentrates were identified by company and industry association representatives as being the link in the 3TG mineral supply chain through which these minerals can be traced to their source of origin (mine); determining the point of origin is critical to establishing a transparent conflict mineral supply chain (U.S. Government Accountability Office, 2012, p. 19). This fact sheet, the first in a series of 3 TG mineral fact sheets, focuses on the tungsten supply chain by listing plants that consume tungsten concentrates to produce ammonium paratungstate and ferrotungsten worldwide.

\section{Background}

The mining and beneficiation of tin (in cassiterite), niobium and tantalum (in columbite-tantalite or "coltan"), tungsten (in wolframite), and gold have been linked to civil unrest and human rights violations in the eastern provinces of the Democratic Republic of the Congo since the late 1990s. During the early 2000s, reports published by the United Nations Group of Experts on the Democratic Republic of the Congo (UN GoE) and other non-governmental organizations identified foreign and domestic armed groups that sought to profit from the production and trade of 3TG minerals. These groups took control of many artisanal mining operations in the eastern Provinces of Katanga, Maniema, North Kivu, Orientale, and South Kivu of the Democratic Republic of the Congo. The revenues collected by these armed groups through the production and sale of 3TG minerals from mining areas under their control reportedly continues to perpetuate civil unrest in the Democratic Republic of the Congo (United Nations Security Council, 2014, p. 3, 42-51).

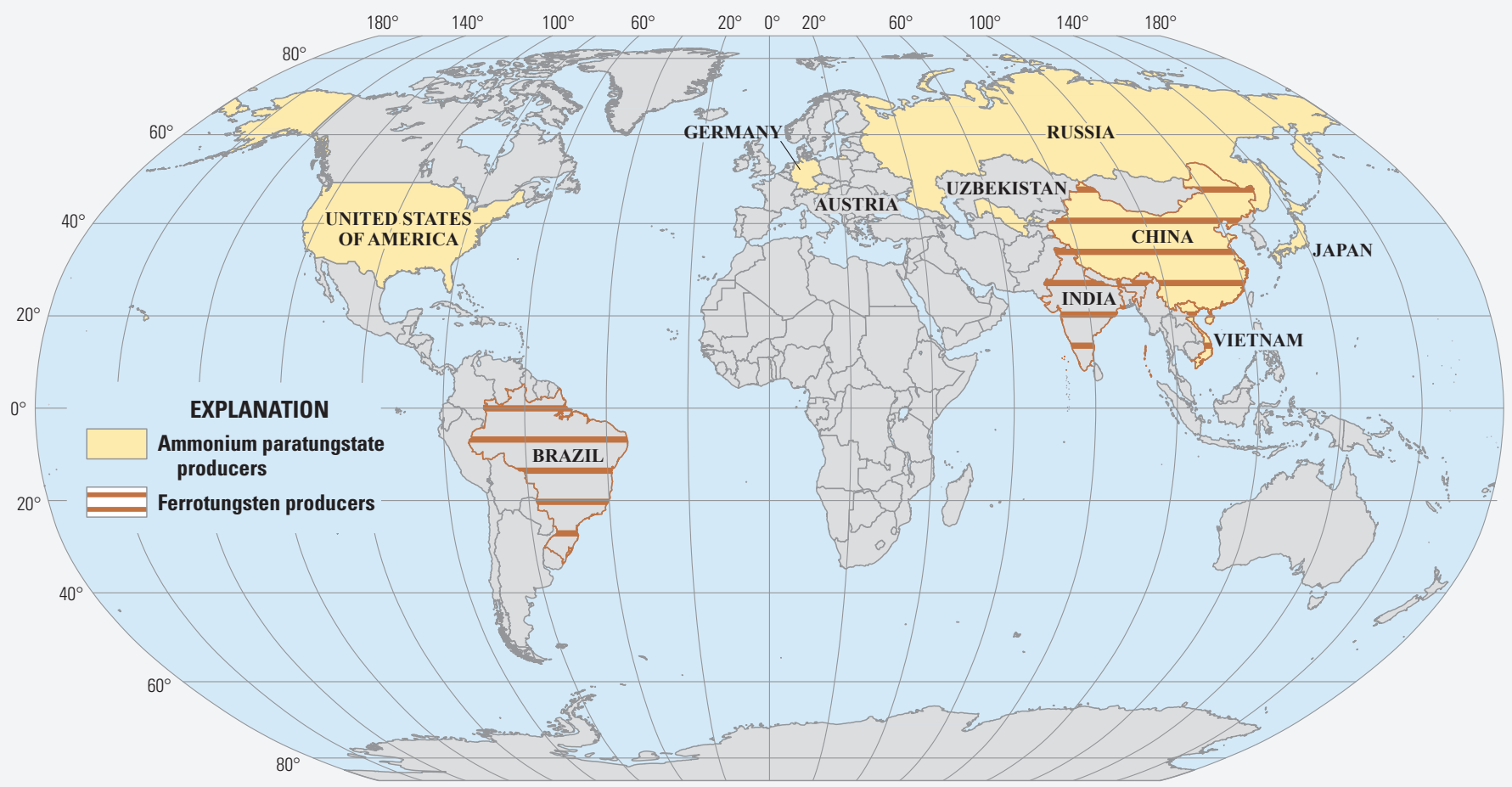

Global consumers of tungsten concentrates for the production of ammonium paratungstate and ferrotungsten in 2013-14. 
Table 1. Tungsten processing plants that consume concentrates.

[APT, Ammonium paratungstate; \%, percent]

\begin{tabular}{|c|c|c|c|}
\hline Country & Plant type & Location & Operator/owner \\
\hline Austria & APT & Bergla, St. Martin im Sulmtal, Styria & Wolfram Bergbau und Hütten AG (Sandvik AB, 100\%) \\
\hline China & APT & Luanchuan County, Henan Province & China Molybdenum Co., Ltd. \\
\hline China & APT & Chongyi County, Ganzhou, Jiangxi Province & Chongyi Zhangyuan Tungsten Co., Ltd. \\
\hline China & APT & $\begin{array}{l}\text { Dayu smelting plant, Dayu County, } \\
\text { Jiangxi Province }\end{array}$ & Dayu Weiliang Tungsten Co., Ltd. \\
\hline China & APT & Dongtai, Jiangsu Province & Dongtai Huihuang Tungsten \& Molybdenum Co., Ltd. \\
\hline China & APT & Longyan, Fujian Province & Fujian Jinxin Tungsten Co., Ltd. \\
\hline China & APT & Fujian Province & Fujian Ninghua Jinjiang Tungsten Co., Ltd. \\
\hline China & APT & Jiangxi Province & Ganxian Shirui New Material Co., Ltd. \\
\hline China & APT & Xincheng & $\begin{array}{l}\text { Ganzhou Haichuang Tungsten Industry Co., Ltd. [Ganzhou } \\
\text { Tejing Tungsten \& Molybdenum Co., Ltd. (GZTJ)] }\end{array}$ \\
\hline China & APT & Ganzhou, Jiangxi Province & $\begin{array}{l}\text { Ganzhou Huaxing Tungsten Products Co., Ltd. [Jiangxi } \\
\text { Tungsten Industry Group Co., Ltd. (JWYX) [joint ven- } \\
\text { ture between China Minmetals and Jiangxi Rare Earth } \\
\text { and Rare Metals Tungsten Group Corp. (JXTC)]] }\end{array}$ \\
\hline China & APT & Zhanggong District, Ganzhou, Jiangxi Province & Ganzhou Non-ferrous Metals Smelting Co., Ltd. \\
\hline China & APT & Ganzhou, Jiangxi Province & $\begin{array}{l}\text { Ganzhou Seadragon W \& Mo Co., Ltd. (Ganzhou Grand } \\
\text { Sea W \& Mo Group Co., Ltd.) }\end{array}$ \\
\hline China & APT & Gan County, Ganzhou, Jiangxi Province & Ganzhou Yuanchi New Material Co., Ltd. \\
\hline China & APT & $\begin{array}{l}\text { Guantang Industrial Zone, Chao'an County, } \\
\text { Chaozhou, Guangdong Province }\end{array}$ & $\begin{array}{l}\text { GuangDong XiangLu Tungsten Co., Ltd. (Chaozhou } \\
\text { Xianglu Tungsten Industry Co., Ltd.) }\end{array}$ \\
\hline China & APT & Ganzhou, Jiangxi Province & $\begin{array}{l}\text { H.C. Starck Jiangwu Tungsten Specialties (Ganzhou) Co., } \\
\text { Ltd. [H.C. Starck GmbH and Jiangxi Rare Metals } \\
\text { Tungsten Holdings Group Co., Ltd. (JXTC)] }\end{array}$ \\
\hline China & APT & $\begin{array}{l}\text { Chenzhou Mining Tungsten Products Plant, } \\
\text { Hunan Province }\end{array}$ & Hunan Chenzhou Mining Group Co., Ltd. \\
\hline China & APT & $\begin{array}{l}\text { Xintang Town, Hengdong County, Hengyang, } \\
\text { Hunan Province }\end{array}$ & $\begin{array}{l}\text { Hunan Chun-chang Non-ferrous Smelting \& } \\
\text { Concentrating Co., Ltd. }\end{array}$ \\
\hline China & APT & $\begin{array}{l}\text { Huanglong Industrial District, Huanglong Town, } \\
\text { Dayu County, Ganzhou, Jiangxi Province }\end{array}$ & Jiangxi Dayu Longxintai Tungsten Co., Ltd. \\
\hline China & APT & $\begin{array}{l}\text { Changlong Town, Chongyi County, Ganzhou, } \\
\text { Jiangxi Province }\end{array}$ & Jiangxi Yaosheng Tungsten Co., Ltd. \\
\hline China & APT & $\begin{array}{l}\text { Langfang Tungsten \& Molybdenum } \\
\text { Material Plant, Langfang, } \\
\text { Hebei Province }\end{array}$ & $\begin{array}{l}\text { Langfang (Torch) Huaying Tungsten \& } \\
\text { Molybdenum Industry Co., Ltd. }\end{array}$ \\
\hline China & APT & $\begin{array}{l}\text { Qiancun, Heyu Town, Luanchuan County, } \\
\text { Luoyang, Henan Province }\end{array}$ & $\begin{array}{l}\text { Luoyang Mudu Tungsten \& Molybdenum } \\
\text { Technology Co., Ltd. }\end{array}$ \\
\hline China & APT & $\begin{array}{l}\text { Xiagou Industry Region, Xigong District, } \\
\text { Luoyang, Henan Province }\end{array}$ & $\begin{array}{l}\text { Luoyang Yongzhuo Tungsten \& Molybdenum } \\
\text { Material Co., Ltd. }\end{array}$ \\
\hline China & APT & Nanchang, Jiangxi Province & Nanchang Cemented Carbide Limited Liability Company \\
\hline China & APT & $\begin{array}{l}\text { Wendeng District, Weihai, Shandong } \\
\text { Province }\end{array}$ & Wendeng Zhengxing Tungsten Industry Co., Ltd. \\
\hline China & APT & $\begin{array}{l}\text { Fujian Xiamen Tungsten Products Plant, } \\
\text { Haicang District, Xiamen, Fujian Province }\end{array}$ & Xiamen Tungsten Co., Ltd. (CXTC) \\
\hline China & APT & $\begin{array}{l}\text { Guihua Village, Renhua County, Shaoguan, } \\
\text { Guangdong Province }\end{array}$ & Xinhai Rendan Shaoguan Tungsten Co., Ltd. \\
\hline China & APT & Hetang District, Zhuzhou, Hunan Province & $\begin{array}{l}\text { Zhuzhou Cemented Carbide Group Co., Ltd. } \\
\text { [Hunan Nonferrous Metals Holding Group (HNG)] }\end{array}$ \\
\hline China & APT & Zigong, Sichuan Province & $\begin{array}{l}\text { Zigong Cemented Carbide Co., Ltd. [Hunan Nonferrous } \\
\text { Metals Holding Group (HNG)] }\end{array}$ \\
\hline
\end{tabular}


Table 1. Tungsten processing plants that consume concentrates.-Continued

[APT, Ammonium paratungstate; \%, percent]

\begin{tabular}{|c|c|c|c|}
\hline Country & Plant type & Location & Operator/owner \\
\hline China & Ferrotungsten & $\begin{array}{l}\text { Sheshan Village, Nanyang Industrial Zone, } \\
\text { Shanghang County, Longyan, Fujian Province }\end{array}$ & $\begin{array}{l}\text { Fujian Ganmin Rare Metal Co., Ltd. [Jiangxi Rare Earth } \\
\text { \& Rare Metals Tungsten Group Corp. (JXTC)] }\end{array}$ \\
\hline China & Ferrotungsten & $\begin{array}{l}\text { Meilin Town, Gan County, Ganzhou, } \\
\text { Jiangxi Province }\end{array}$ & Ganzhou BESEEM Ferrotungsten Co., Ltd. \\
\hline China & Ferrotungsten & Ganzhou, Jiangxi Province & $\begin{array}{l}\text { Ganzhou Jiangwu Ferrotungsten Co., Ltd. [Jiangxi Tung- } \\
\text { sten Industry Group Co., Ltd. (JWYX) [joint venture } \\
\text { between China Minmetals and Jiangxi Rare Earth and } \\
\text { Rare Metals Tungsten Group Corp. (JXTC)]] }\end{array}$ \\
\hline China & Ferrotungsten & $\begin{array}{l}\text { Wu Town, Hengdong County, Hengyang, } \\
\text { Hunan Province }\end{array}$ & Hunan Chuangda Metallurgy Group Co., Ltd. \\
\hline China & Ferrotungsten & Ganzhou, Jiangxi Province & $\begin{array}{l}\text { Jiangxi Richsea New Material Co., Ltd. (Ganzhou } \\
\text { Grand Sea W \& Mo Group Co., Ltd.) }\end{array}$ \\
\hline China & Ferrotungsten & $\begin{array}{l}\text { Xinxiangxilu, Xiangxiang, Xiangtan, } \\
\text { Hunan Province }\end{array}$ & Minmetals (Hunan) Ferroalloys Co., Ltd. \\
\hline China & Ferrotungsten & $\begin{array}{l}\text { Jiuli Township, Emeishan City, Leshan, } \\
\text { Sichuan Province }\end{array}$ & $\begin{array}{l}\text { Chuantou Emei Ferroalloy [Sichuan Chuantou } \\
\text { Emei Ferroalloy (Group) Co., Ltd.] }\end{array}$ \\
\hline China & Ferrotungsten & Jilin, Jilin Province & Sinosteel Jilin Ferroalloy Corporation, Ltd. \\
\hline Germany & APT & Im Schleeke, Goslar, Lower Saxony & H.C. Starck GmbH \\
\hline India & Ferrotungsten & Churchgate, Mumbai, Maharashtra State & Bharat Pulverising Mills Ltd. \\
\hline India & Ferrotungsten & SDKC Unit, Verka, Amritsar, Punjab & Mehra Ferro-Alloys Pvt. Ltd. \\
\hline India & Ferrotungsten & Thane-Belapur, Mumbai, Maharashtra State & Sunbel Alloys Co. of India Ltd. \\
\hline India & Ferrotungsten & Rairangpur, Mayurbhanj District, Odisha State & Superb-Metal Alloys (Pvt.) Ltd. \\
\hline Japan & APT & Akita, Akita Prefecture & Japan New Metals Co., Ltd. \\
\hline Russia & APT & Nalchik, Kabardino-Balkariya Republic & JSC Hydrometallurg (Wolfram Company CJSC) \\
\hline Russia & APT & $\begin{array}{l}\text { Kirovgrad Hard Alloys Plant, Kirovgrad, } \\
\text { Sverdlovskaya Oblast }\end{array}$ & JSC KZTS \\
\hline Russia & APT & Vladikavkaz, North Ossetia Republic & JSC Pobedit (Wolfram Company CJSC) \\
\hline United States & APT & Towanda, Pennsylvania & Global Tungsten \& Powders Corp. \\
\hline United States & APT & Huntsville, Alabama & Kennametal, Inc. (previously ATI Alldyne) \\
\hline United States & APT & Depew, New York & $\begin{array}{l}\text { Niagara Refining LLC (Buffalo Tungsten Inc. } \\
\text { and Sumitomo Electric Industries, Ltd.) }\end{array}$ \\
\hline United States & $\begin{array}{l}\text { Tungsten carbide } \\
\text { producer that } \\
\text { consumes } \\
\text { concentrate }\end{array}$ & Fallon, Nevada & Kennametal, Inc. \\
\hline Uzbekistan & APT & Chirchiq, Tashkent Province & $\begin{array}{l}\text { Uzbek Refractory and Heat-Resistant Metals (OJSC } \\
\text { "UzKTJM") }\end{array}$ \\
\hline Vietnam & APT & Dai Tu District, Thai Nguyen Province & Nui Phao Mining Company Ltd. and H.C. Starck GmbH \\
\hline Vietnam & APT & Nhon Trach District, Dong Nai Province & Sanher Tungsten Vietnam Co., Ltd. \\
\hline Vietnam & APT & Trang Bang District, TayNinh Province & Tejing (Vietnam) Tungsten Co., Ltd. \\
\hline Vietnam & APT & $\begin{array}{l}\text { Cailan Industrial Zone, Halong City, } \\
\text { Quang Ninh Province }\end{array}$ & Vietnam Youngsun Tungsten Industry Co., Ltd. \\
\hline Vietnam & Ferrotungsten & $\begin{array}{l}\text { Tan Tien Industrial Zone, Vinh Bao District, } \\
\text { Hai Phong }\end{array}$ & $\begin{array}{l}\text { ATC Ferrotungsten [Asia Tungsten Products } \\
\text { Vietnam Ltd. (ATC)] }\end{array}$ \\
\hline Vietnam & Ferrotungsten & $\begin{array}{l}\text { Cailan Industrial Zone, Halong City, } \\
\text { Quang Ninh Province }\end{array}$ & Vietnam Youngsun Tungsten Industry Co., Ltd. \\
\hline
\end{tabular}


The international community, through a multistakeholder process, which included the International Conference on the Great Lakes Region (ICGLR), the Organisation for Economic Co-operation and Development (OECD), and the UN GoE, has responded by developing voluntary due diligence guidance for minerals from conflict-affected and high-risk areas. This guidance helps companies avoid inadvertently contributing to conflict and human rights abuses in the Democratic Republic of the Congo and the Great Lakes Region of Africa (Organisation for Economic Co-operation and Development, 2013, p. 3). The United States responded to the situation in the Democratic Republic of the Congo by enacting Section 1502 of the Dodd-Frank Wall Street Reform and Consumer Protection Act (Dodd-Frank Act) (Pub. L. 111-203, 124 Stat. 1376-2223) on July 21, 2010, which requires companies that file annual reports with the Securities and Exchange Commission (SEC) to publicly disclose due diligence measures to determine if $3 \mathrm{TG}$ minerals were sourced from the Democratic Republic of the Congo or an adjoining country (defined as a country that shares an internationally recognized border with the Democratic Republic of Congo), and whether these minerals benefitted armed groups or abusive armed forces within the Africa Great Lakes Region. If the minerals are "necessary to the functionality or production of a product" manufactured by those firms, then businesses must undertake a "country of origin" inquiry to determine their source. If the minerals are, or are suspected to be, sourced from countries specified in the Dodd-Frank Act, then firms must conduct due diligence on their supply chains in conformance with internationally accepted frameworks, such as OECD's guidance (U.S. Securities and Exchange Commission, 2010, p. 842; 2014).

Under Section 1502 of the Dodd-Frank Act, companies must file a specialized disclosure form (SD Form) with the SEC that includes their findings and the opinion of an independent auditor indicating whether or not any $3 \mathrm{TG}$ minerals used in their products and components are sourced from countries specified in the Dodd-Frank Act. If a company determines that their products or components include 3TG minerals from any of these countries, it is required to trace those minerals back to the mine of origin, often through a complex chainof-custody that may include multiple component manufacturers, processing facilities (smelters and refineries), intermediaries, shipping docks, and trade centers (U.S. Securities and Exchange Commission, 2014). The first deadline for companies required to file an SD Form with the SEC was June 2, 2014.

\section{Tungsten Processing Facility Data}

Table 1 displays more than 50 mineral processing facilities that consume tungsten concentrates, and includes available data regarding the name, location, processing plant type, and ownership. The USGS, however, does not have complete data on the source of origin of the concentrates used by these processing plants and therefore cannot verify whether these facilities consumed concentrates from either conflict-affected and high-risk areas, the Democratic Republic of the Congo, or an adjoining country. The information in this table represents plants that could have processed tungsten concentrates in 2013-14. The table does not include plants that only process recycled materials or that only process downstream materials made from concentrates and (or) scrap.

The data were evaluated and compiled by the USGS National Minerals Information Center from sources that include companies, foreign governments, industry analysts, industry associations, inter-governmental organizations, non-governmental organizations, and trade journals. While every attempt was made to include all concentrate-consuming plants, some plants were not listed. For example, information was not available on all tungsten processing plants in China, and Brazil is thought to produce ferrotungsten, but information on specific plants could not be confirmed.

ISSN 2327-6916 (print)

ISSN 2327-6932 (online)

http://dx.doi.org/10.3133/fs20143069

\section{References Cited}

Organisation for Economic Co-operation and Development (OECD), 2013, OECD due diligence guidance for responsible supply chains of minerals from conflict-affected and high-risk areas (Second edition): Paris, OECD, 118 p. [Also available at $h t t p: / / d x . d o i$. org/10.1787/9789264185050-en.]

U.S. Government Accountability Office (GAO), 2012, Report to Congressional committees - Conflict minerals disclosure ruleSEC's actions and stakeholder-developed initiatives: Washington, D.C., U.S. GAO, Report GAO-12-763, 45 p. [Also available at http://www.gao.gov/assets/600/592458.pdf.]

U.S. Securities and Exchange Commission (SEC), 2010, One Hundred Eleventh Congress of the United States of America - Dodd-Frank Wall Street Reform and Consumer Protection Act: Washington, D.C., U.S. SEC, 848 p. (Accessed June 7, 2013, at: http://www.sec.gov/ about/laws/wallstreet reform-cpa.pdf.)

U.S. Securities and Exchange Commission (SEC), 2014, Fact SheetDisclosing the use of conflict minerals: Washington, D.C., U.S. SEC, accessed July 3, 2014, at http://www.sec.gov/News/Article/ Detail/Article/1365171562058.

United Nations Security Council, 2014, Letter dated 22 January 2014 from the coordinator of the Group of Experts on the Democratic Republic of the Congo addressed to the President of the Security Council: New York, United Nations Security Council, no. 14-21515, 277 p. [Also available at $h t t p: / /$ www.securitycouncilreport.org/atf/cf/\%7B65BFCF9B-6D27-4E9C8CD3-CF6E4FF96FF9\%7D/s_2014_42.pdf.]

\section{Information Contacts}

Steven D. Textoris

Chief, Global Minerals Analysis Section

National Minerals Information Center

U.S. Geological Survey

991 National Center

Reston, VA 20192

stextoris@usgs.gov

http://minerals.usgs.gov/minerals

Omayra Bermúdez-Lugo

Country Specialist - West Africa, Angola,

Central African Republic, Namibia, and

The Indian Ocean Islands

Global Minerals Analysis Section

National Minerals Information Center, U.S. Geological Survey obermude@usgs.gov

Kim B. Shedd

Mineral Commodity Specialist - Cobalt and Tungsten

Mineral Commodities Section

National Minerals Information Center, U.S. Geological Survey

kshedd@usgs.gov

Donald I. Bleiwas

Physical Scientist

Global Minerals Analysis Section

National Minerals Information Center, U.S. Geological Survey bleiwas@usgs.gov

Thomas R. Yager

Country Specialist-East and Southern Africa

Global Minerals Analysis Section

National Minerals Information Center, U.S. Geological Survey tyager@usgs.gov 